\title{
Traitement des alvéolites post-extractionnelles. Données actuelles et expérience du centre de consultations et de traitements odonto-stomatologiques (CCTOS) d'Abidjan
}

\section{Treatment of postextraction alveolitis. Current informations and experience of the dental and stomatological treatment center of Abibjan}

\author{
KOUAKOU SOUAGA ${ }^{1,3}$, PATRICE ATTOBGAIN KOUAME ${ }^{1,3}$, JEANNETTE AKA-ADOUKO ${ }^{1,3}$,
} AKPE JONAS ADOU ${ }^{2,3}$, AKAII ADOU $^{1,3}$, YAPO ANGOH ${ }^{1,3}$

\begin{abstract}
RÉSUMÉ
Les alvéolites post-extractionnelles sont les complications les plus fréquentes des extractions dentaires. Elles sont ubiquitaires. Leur étiopathogénie n'est pas totalement élucidée et leur traitement diffère selon les auteurs. Cet article comporte, dans une première partie, une revue de la littérature axée sur les aspects étio-pathogénique, symptomatologique et thérapeutique de ces complications post-extractionnelles. Dans une deuxième partie, les auteurs font part de leur expérience dans le traitement de ces affections. Ils utilisent deux protocoles : le premier repose sur l'emploi d'une pâte extemporanée préparée à partir d'une association antibiotique (bacitracine + néomycine) et d'eugénol, le second est basé sur l'utilisation en pansements intra-alvéolaires de ciprofloxacine sous sa présentation de gouttes auriculaires.

En raison de la facilité de leur mise en œuvre et des résultats obtenus, les auteurs recommandent l'utilisation de ces protocoles en cas d'alvéolites post-extractionnelles. (Med Buccale Chir Buccale 2009 ; 15: 147-151).
\end{abstract}

médecine buccale chirurgie buccale VOL. $15, \mathrm{~N}^{\circ} 3$ 2009 page 147

mots clés: alvéolite, extraction dentaire, bacitracine, néomycine, ciprofloxacine

\section{SUMMARY}

The alveolitis are the most common complications after dental extractions. Their aetiology and pathogenesis are not completely established. Their treatment differs according to the authors.

In a first part, this article makes a review of the literature about the aetio-pathogenical, symptomatological and therapeutical aspects of these post-extraction complications. In a second part, the autors announce their experience in the treatment of these affections. They present two protocols. In the first, they use an association of antibiotic (bacitracin+neomycin) and eugenol paste. The second is based on using of alveolar dressing with auricular drops of ciprofloxacin.

Because of their easy use and the results obtained, the authors recommend their protocols in the event of postextractionnal alveolitis. (Med Buccale Chir Buccale 2009 ; 15: 147-151).

key words: alveolitis, dental extraction, bacitracin, neomycin, ciprofloxacin

\footnotetext{
1. Service de Chirurgie Pathologie et Thérapeutique Centre de Consultations et de Traitements odonto-stomatologiques (CCTOS) CHU Cocody Abidjan Côte d'Ivoire

2. Service d'Odontologie Conservatrice-Endodontie CHU Cocody Abidjan Côte d'Ivoire

3. UFR d'Odonto-Stomatologie Université Cocody Abidjan Côte d'Ivoire
}

Demande de tirés à part:

Souaga Kouakou 22 BP 1231 Abidjan 22 swagat3@yahoo.fr 
médecine

page 148
Les alvéolites post-extractionnelles sont dues à une inflammation de la paroi alvéolaire qui survient après les extractions dentaires. II s'agit d'ostéites aiguës circonscrites de l'os alvéolaire. Ce sont les complications les plus fréquentes des extractions dentaires : elles surviennent dans 1 à $3 \%$ des cas après les extractions simples $[1,2,3]$ et dans 5 à $35 \%$ des cas après les extractions chirurgicales ${ }^{[1,3,4]}$.

Elles sont pénibles pour les patients dans la mesure où les douleurs qu'elles induisent sont rebelles aux antalgiques de palier I selon la classification de l'OMS. Elles sont embarrassantes pour les praticiens qui revoient les patients en urgence (donc plutôt que prévu) et qui sont tenus pour responsables.

Leur traitement a fait l'objet de plusieurs publications. Cela atteste de la pertinence de ces pathologies et de la diversité de leur prise en charge. En effet les protocoles diffèrent selon les auteurs. Nous faisons part de l'expérience de notre service après un point sur les données actuelles. Celles-ci sont relatives à l'étiopathologie, à la symptomatologie et aux traitements proposés actuellement.

\section{DONNÉES ACTUELLES}

\section{Etiopathogénie}

On distingue trois formes d'alvéolites : sèche, suppurée et ostéitique parcellaire (ou cellulite du $21^{\mathrm{e}}$ jour) [5].

L'alvéolite sèche ou dry socket est la plus fréquente d'entre elles et partant, des complications post-extractionnelles [6, 7, 8, 9]. La majeure partie des études a été consacrée à cette forme. La pathogénie de cette forme d'alvéolite n'est pas totalement élucidée. A ce jour, trois théories sont avancées pour expliquer sa survenue. Ce sont : - l'absence de formation de caillot par insuffisance d'apport sanguin péri-alvéolaire, notamment à la mandibule où la vascularisation est de type terminal ;

- la malformation du caillot suite au traumatisme consécutif à l'extraction dentaire ;

- la lyse du caillot, qui est la théorie la plus partagée.

Elle repose sur l'augmentation de la fibrinolyse au sein de l'alvéole sans qu'il y ait pour autant une augmentation de la fibrinolyse systémique ou salivaire [10]. La fibrinolyse est due à la plasmine formée à partir de l'activation du plasminogène. On distingue les activateurs intrinsèques (ou directs) des activateurs extrinsèques (ou indirects). Les premiers sont représentés par des enzymes de conversion que l'on retrouve dans des tissus aussi divers que la muqueuse buccale, le périoste, la moelle osseuse, etc. Les seconds sont représentés par le traumatisme osseux engendré par l'extraction, les microorganismes de la cavité buccale (le plus suspecté étant Treponema denticola car il a une forte activité « plasmin like ») et les agents chimiques tels que les anti-inflammatoires, les contraceptifs oraux et le tabac [11]. L'alvéolite sèche est donc une complication multifactorielle. Aguirre [12] a décrit le sujet type pour développer cette forme d'alvéolite : sujet de sexe féminin, âgé de 30 à 50 ans, stressé, sous contraceptif oral, à hygiène bucco-dentaire moyenne ou mauvaise et dont la dent à extraire est une molaire mandibulaire sur l'arcade.

L'alvéolite suppurée est consécutive à la surinfection de l'alvéole ou du caillot. Elle est favorisée par le manque d'asepsie et la présence de corps étrangers (débris osseux, dentaires ou tartriques intra-alvéolaires). Les infections préexistantes ou postérieures à l'extraction, l'extension de l'infection à partir des dents adjacentes ainsi que l'insuffisance d'hygiène bucco-dentaire constituent également des facteurs favorisants. L'alvéolite ostéitique parcellaire ou cellulite du $21^{\mathrm{e}}$ jour est due à une surinfection du tissu de granulation. Elle a la particularité d'apparaître vers la $3^{\mathrm{e}}$ semaine ( $21^{\mathrm{e}}$ jour, d'où son nom) suivant l'extraction dentaire. Pour Le Toux [13], la cellulite du $21^{\mathrm{e}}$ jour serait une complication spécifique à la germectomie des dents de sagesse mandibulaires. Elle est favorisée par la présence de corps étrangers (débris alimentaires, sac péricoronaire insuffisamment cureté, séquestre osseux) ou par la prise inadaptée d'anti-inflammatoires non stéroïdiens (AINS) après l'intervention chirurgicale.

\section{Symptomatologie}

L'alvéolite sèche survient quelques heures à cinq jours après l'extraction dentaire. Les signes cliniques de cette forme ont été décrits pour la pre- 
mière fois en 1886, par Crawford. Les signes généraux sont généralement discrets : fébricule et légère asthénie. Les signes fonctionnels sont marqués par des douleurs d'intensité variable. II peut s'agir de petits épisodes douloureux, intermittents avec irradiation vers l'oreille ou l'hémiface. Cependant, le plus souvent, il s'agit de douleurs intenses, continues, de plus en plus insensibles aux antalgiques de niveau I, voire de niveau II. Elles sont exacerbées par le décubitus, ce qui rend l'insomnie quasi-constante dans cette forme d'alvéolite ${ }^{[3]}$. II existe souvent une halitose. Les signes physiques sont dominés par la vacuité de l'alvéole dont les parois sont blanc grisâtre et très sensibles au toucher. La muqueuse périalvéolaire est inflammatoire. L'écouvillonnage de l'alvéole met en évidence une odeur fétide. L'examen radiographique ne révèle rien de particulier, le plus souvent. L'évolution spontanée de l'alvéolite sèche se fait généralement vers la guérison au bout d'une dizaine de jours. En cas de traitement, ce délai est écourté. Toutefois, il est à craindre au cours de cette évolution spontanée, des complications à type d'ostéite plus étendue. L'alvéolite suppurée survient en moyenne cinq jours après l'extraction. Les signes généraux se résument à une fièvre de 38 à $38,5^{\circ} \mathrm{C}$. Les signes fonctionnels sont constitués par des douleurs moins intenses que celles de l'alvéolite sèche ; elles sont sourdes et pulsatiles. Les signes physiques sont marqués par une adénopathie satellite, une tuméfaction vestibulaire associée ou non à une fistule muqueuse péri-alvéolaire, une alvéole comblée par un caillot de couleur brunâtre ou noirâtre saignotant ou laissant sourdre du pus fétide. Les parois alvéolaires sont très sensibles. Des débris osseux, dentaires ou tartriques sont souvent retrouvés au fond de l'alvéole. L'évolution est non résolutive spontanément : elle se fait vers des complications à type d'alvéolite ostéitique parcellaire ${ }^{[4]}$.

L'alvéolite ostéitique parcellaire, comme la forme suppurée, se traduit par une fièvre de 38 à $38,5^{\circ} \mathrm{C}$. Les signes fonctionnels sont ici représentés par des douleurs persistantes depuis environ 15 jours. Un trismus peut être associé. Les signes physiques sont marqués par une asymétrie faciale due à une cellulite péri-mandibulaire associée, un comblement du vestibule et la présence ou non d'une fistule cutanée. La radiographie met le plus souvent en évidence un séquestre osseux, mais parfois elle ne révèle rien. L'évolution en l'absence de traitement se fait vers l'élimination du séquestre ou vers des complications infectieuses plus graves.

\section{Traitement}

Il a pour but de permettre à la cicatrisation de l'alvéole de reprendre son cours normal. II peut être préventif et curatif. Le traitement préventif repose essentiellement sur:

- l'élimination des facteurs étiologiques,

- un bilan pré-extractionnel complet,

- une préparation adéquate à l'intervention, tant sur le plan local que sur le plan général,

- la réalisation des extractions dentaires selon les règles de l'art,

- la motivation des patients à maintenir une hygiène bucco-dentaire correcte et au respect des soins et des recommandations post-extractionnelles.

Le traitement curatif est fonction des formes cliniques. Celui des alvéolites suppurée et ostéitique parcellaire est relativement bien codifié. II est basé sur une antibiothérapie (par voie générale), une antalgie et des soins locaux (révision alvéolaire, rinçage à l'aide d'une solution saline ou antiseptique et pansements intra-alvéolaires). Concernant l'alvéolite suppurée Perrin et coll. [3] précisent que si les soins locaux sont réalisés précocement et en l'absence de signes généraux, la prescription d'antibiotique n'est pas nécessaire. En ce qui concerne le traitement de l'alvéolite sèche, il relève de l'habitude de chaque praticien. Plusieurs antibiotiques utilisés seuls ou associés à diverses autres substances ont été proposés pour le traitement de ces complications [14-16]. L'utilisation de pansements intra-alvéolaires à base d'antibiotique a déjà été suggérée dans le traitement des alvéolites ${ }^{[16,17]}$. La tétracycline et la clindamycine sont les deux molécules les plus recommandées $[11,15,17]$.

Dans le but de contribuer au traitement des alvéolites, nous présentons notre expérience. \begin{tabular}{l}
$\begin{array}{l}\text { médecine } \\
\text { buccale } \\
\text { chirurgie } \\
\text { buccale }\end{array}$ \\
\hline voL. $15, N^{\circ} 3$ \\
2009
\end{tabular}

page 149 


\section{L'EXPERIENCE DU CENTRE DE CONSULTATIONS ET DE TRAITEMENTS ODONTO-STOMATOLOGIQUES (CCTOS) D'ABIDJAN}

Depuis plus d'une dizaine d'années, nous utilisons deux protocoles.

\section{Protocole $n^{\circ} 1$}

II repose sur l'utilisation de pansements intra-alvéolaires préparés extemporanément à partir de l'association bacitracine-néomycine (Baneocin ${ }^{\circledR}$ poudre dermique) et d'eugénol.

Il débute par un bain de bouche que le patient effectue à l'aide d'une solution d'hexétidine ou de chlorhexidine, pendant 30 à 40 secondes. Puis, une anesthésie sans vaso-constricteur est réalisée à distance du site de l'alvéolite. Dès que l'anesthésie est effective, une révision alvéolaire médecine buccale chirurgie buccale

page 150 à la curette est effectuée afin d'obtenir un nouveau caillot à même de combler l'alvéole, donc de protéger les parois de celle-ci. Dès l'obtention d'un saignement intra-alvéolaire suffisant, l'hémostase est réalisée à l'aide d'une compresse de gaze stérile maintenue en occlusion par le patient pendant deux à cinq minutes. Pendant ce temps, on prépare sur une plaque de verre, une pâte composée des antibiotiques en poudre et de l'eugénol. Celle-ci doit avoir la consistance d'un morceau de sucre mouillé, toute la poudre utilisée doit être enrobée par l'eugénol. L'alvéole est isolée de la salive et comblée, sans tassement, par la pâte ainsi obtenue à l'aide d'une spatule à bouche. Le pansement ainsi réalisé est maintenu en place grâce à une compresse de gaze stérile sur laquelle il est demandé au patient de mordre pendant une trentaine de minutes. Des bains de bouche et un antalgique non anti-inflammatoire de niveau I (généralement du paracétamol) sont prescrits. Il est recommandé au patient de débuter les bains de bouche seulement 12 heures après la mise en place du pansement. Enfin le patient est revu tous les jours ou tous les deux pour un contrôle et un renouvellement du pansement. La durée du traitement varie généralement de 7 à 10 jours.

\section{Protocole $\mathrm{n}^{\circ} 2$}

Il est basé sur l'utilisation de la ciprofloxacine sous sa présentation de gouttes auriculaires (Cetraxal Otico ${ }^{\circledR}$ ) en pansements intra-alvéolaires. Les étapes préliminaires sont identiques à celles du protocole $\mathrm{n}^{\circ} 1$. Après l'hémostase, 6 ou 8 gouttes sont déposées dans l'alvéole. Les dimensions de celle-ci déterminent le nombre de gouttes à instiller. Le même type de prescriptions médicamenteuses est fait et les mêmes recommandations formulées. II en est de même pour la fréquence du renouvellement des instillations et pour la durée du traitement.

Quel que soit le protocole mis en œuvre, les résultats cliniques obtenus sont superposables, c'està-dire :

- disparition des douleurs spontanées et de l'hypersensibilité des parois alvéolaires dès le deuxième jour,

- reprise de la cicatrisation dès le quatrième jour,

- obtention d'une cicatrisation alvéolaire satis-

faisante après sept jours de traitement.

Les résultats sont spectaculaires au point que plusieurs malades sont perdus de vue dès le troisième ou le quatrième pansement. Ce n'est que longtemps après que certains d'entre eux sont revus pour un autre motif de consultation ou dans un tout autre cadre.

Contrairement aux pansements prêts à l'emploi où les risques de contamination augmentent avec le temps, nos protocoles minimisent ces risques. En effet, la poudre associant bacitracine-néomycine et les gouttes de ciprofloxacine sont contenues dans des flacons qui permettent une utilisation sans exposer le produit restant.

Nos protocoles sont de mise en œuvre facile ; parfois même, les patients compliants font les pansements à la ciprofloxacine, à domicile. L'utilisation de nos protocoles est subordonnée à l'absence de contre-indications à la bacitracine, à la néomycine ou à la ciprofloxacine.

$\mathrm{Ni}$ l'association bacitracine-néomycine ni la ciprofloxacine n'ont d'AMM pour les alvéolites postextractionnelles. Cette situation n'est pas exceptionnelle. En effet, il existe plusieurs autres exemples dans l'histoire de la médecine. On peut en rappeler deux biens connus. Les imidazolés notamment le métronidazole, ont été initialement 
commercialisés comme antiparasitaires. Très rapidement l'usage empirique a permis de découvrir leur efficacité sur les anaérobies. Ils sont prescrits beaucoup plus comme agents anti-anaérobies que comme antiparasitaires. De même, la colchicine est indiquée pour le traitement de l'accès aigu de goutte, des arthrites pseudo-goutteuses et de la maladie périodique ${ }^{[18]}$. On sait maintenant qu'elle constitue un traitement efficace contre l'aphtose buccale récidivante [19,20].

La quasi-totalité des alvéolites traitée dans le service, est de forme sèche. Aucun cas d'alvéolite ostéitique parcellaire n'a été observé. Quelques rares cas d'alvéolites suppurées (à leur début) ont été observées et ont également motivé l'utilisation de l'un des deux protocoles.

Aucun effet indésirable n'a été observé ou signalé par nos patients.

\section{RÉFÉRENCES}

1 - Horch H. Chirurgie buccale. Masson, Paris 1996.

2 - Larsen PE. Alveolar osteitis after removal of impacted mandibular third molars. Oral Surg Oral Med Oral Pathol $1992 ; 73: 393-7$.

3 - Perrin D, Ahossi V, Larras P, Paris M. Douleur. In: L'urgence en odontologie (pp 23-38). CdP, Paris, 2005

4 - Taiarol L, Huet P, Schmidt J, Mercier J. Alvéolites et complications infectieuses aiguës après extractions des dents de sagesse. Cah Stomatol Chir Maxillofac 1996 ; $3: 54-7$.

5 - Goudaert M, Danhiez P Dictionnaire pratique d'odontologie et de stomatologie. Masson, Paris, 1983

6 - Bloomer CR. Alveolar osteitis prevention by immediate placement of medicated packing. Oral Surg Oral Med Oral Pathol Oral Radiol Endod 2000 ; 90 : 282-4.

7 - Sanchis Jm, Penarrocha M, Gay C. Tetracycline compound placement to prevent dry socket: a postoperative study of 200 impacted mandibular third molars. J Oral Maxillofac Surg 2004 ; 62 : 587-91.

8 - Sorensen DC, Preisch JW. The effect of tetracycline on the incidence of postextraction alveolar osteitis. J Oral Maxillofac Surg 1987 ; 45 : 1029-33.

9 - Vezeau PJ. Dental extraction wound management : medicating postextracttion sockets. J Oral Maxillofac Surg $2000 ; 58: 531-7$.

10 - Awang MN. The aetiology of dry socket: a review. Int Dent J 1989 ; 236-40.

\section{CONCLUSION}

Les alvéolites sont les complications post-extractionnelles les plus fréquentes. Elles sont douloureuses et embarrassantes : elles font partie des urgences en odontologie. Leur traitement est plus ou moins codifié selon les formes. L'alvéolite sèche qui est de loin la plus fréquente, a fait l'objet de plusieurs protocoles. Nous avons l'expérience de pansements intra-alvéolaires à base soit d'une association de bacitracine-néomycine et d'eugénol soit de ciprofloxacine sous sa forme de gouttes auriculaires. En raison des bons résultats obtenus avec ces deux protocoles, nous proposons leur utilisation en cas d'alvéolites post-extractionnelles.

11 - Lambert S, Reychler H. L'alvéolite sèche. Prévention et traitement. Rev Stomatol Chir Maxillofac 1994 ; 95 : 435-40.

12 - Aguirre R. Les alvéolites post-extractionnelles : qu'en est-il en 2003 ? Thèse de doctorat en Chirurgie dentaire, $n^{\circ} 17$, Bordeaux 2003.

13 - Le Toux G. Complications infectieuses des germectomies de dents de sagesse mandibulaires. La cellulite du $21^{\mathrm{e}}$ jour. Chir Dent Fr 1994, 683/684 : 69-72.

14 - Souaga K, Adou A, Amantchi D, Angoh Y. Traitement des alvéolites en pratique odonto-stomatologique. Notre expérience. Odontostomatol Trop 1997 ; 20 : 21-5.

15 - Swanson AE. A double blind study of the effectiveness of tetracycline in reducing the incidence of fibrinolytic alveolitis. J Oral Maxillofac Surg 1989 ; 47 : 165-7.

16 - Krekmanov L. Alveolitis after operative removal of third molars in the mandibule. Int J Oral Surg 1981 ; 10 : 1739.

17 - Swanson AE. Prevention of dry socket, an overview. Oral Surg Oral Med Oral Pathol 1990 ; 70 : 131-6.

18 - Vidal 2006

19 - Fontes V, Machet L, Huttenberger B, Lorette G, Vaillant $L$. Aphtose buccale récidivante : traitement par colchicine. Etude ouverte de 54 cas. Ann Dermatol Venereol $2002 ; 129$ : 1365-9.

20 - Ben Slama L, Djemil M. La colchicine. Rev Stomatol Chir Maxillofac 2002 ; 103 : 128-9. médecine buccale chirurgie buccale VOL. $15, \mathrm{~N}^{\circ} 3$ 2009 page 151 